\title{
ON A PROBLEM OF GRAPH THEORY
}

by

P. ERDŌS', A. RÉNYI ${ }^{2}$ and V. T. SOS 2

\section{§. Introduction}

Let $G_{n}$ be a non-directed graph having $n$ vertices, without parallel edges and slings. Let the vertices of $G_{n}$ be denoted by $P_{1}, \ldots, P_{n}$. Let $v\left(P_{i}\right)$ denote the valency of the point $P_{i}$ and put

$$
V\left(G_{n}\right)=\max _{1 \pm i=n} v\left(P_{i}\right) .
$$

Let $E\left(G_{n}\right)$ denote the number of edges of $G_{n}$. Let $\mathbf{H}_{a}(n, k)$ denote the set of all graphs $G_{n}$ for which $V\left(G_{n}\right)=k$ and the diameter $D\left(G_{n}\right)$ of which is $\leqq d$, $(k=1,2, \ldots, n-1 ; d=2,3, \ldots, n-1)$.

In the present paper we shall investigate the quantity

$$
F_{d}(n, k)=\min _{G_{n} \in H_{d}(n, k)} E\left(G_{n}\right) .
$$

Thus we want to determine the minimal number $N$ such that there exists a graph having $n$ vertices, $N$ edges and diameter $\cong d$ and the maximum of the valencies of the vertices of the graph is equal to $k$.

To help the understanding of the problem let us consider the following interpretation. Let be given in a country $n$ airports; suppose we want to plan a network of direct flights between these airports so that the maximal number of airports to which a given airport can be connected by a direct flight should be equal to $k$ (i.e. the maximum of the capacities of the airports is prescribed), further it should be possible to fly from every airport to any other by changing the plane at most $d-1$ times; what is the minimal number of flights by which such a plan can be realized? For instance, if $n=7, k=3, d=2$ we have $F_{2}(7,3)=9$

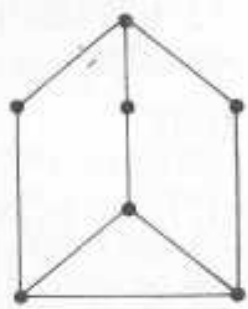

Fig. 1 and the extremal graph is shown by Fig. 1.

The problem of determining $F_{d}(n, k)$ has been proposed and discussed recently by two of the authors (see [1]). In $\$ 1$ we give a short summary of the results of the paper [1], while in $\$ 2$ and 3 we give some new results which go beyond those of [1]. Incidentally we solve a long-standing problem about the maximal number of edges of a graph not containing a cycle of length 4 .

In $\$ 4$ we mention some unsolved problems.

Let us mention that our problem can be formulated also in terms of $0-1$ matrices as follows: Let $M=\left(\varepsilon_{i j}\right)$ be a symmetrical $n$ by $n$ zero-one matrix such

" Mathematical Institute of the Hungarian Academy of Sciences.

${ }^{2}$ Eötvös L. University, Budapest. 
that $\varepsilon_{i i}=1, \quad \max _{1 \leq i \leq n} \sum_{j=1}^{n} \varepsilon_{i j}=k+1$ and all elements of $M^{d}$ are $\equiv 1$. We want to determine

Clearly

$$
M_{d}(n, k)=\min \sum_{i=1}^{n} \sum_{j=1}^{n} \varepsilon_{i j} .
$$

$$
M_{d}(n, k)=2 F_{d}(n, k)+n
$$

This formulation shows the connection of our problem with non-linear programming.

We give for the case $d=2$ a third formulation of our problem which displays its connection with the theory of block designs,

Let be given a sequence $A_{1}, A_{2}, \ldots, A_{n}$ of subsets of the elements $1,2, \ldots, n$ such that if $j \in A_{i}$ then $i \in A_{j}$. Let us suppose that denoting by $|A|$ the cardinal number of the set $A$, we have $\max _{1 \rightarrow j \leq n}\left|A_{j}\right|=k$. Let us suppose that for any $i(1 \leqq i \leqq n)$ and any $j \neq i$ such that $j \& A_{i}$ there is a set $A_{k}$ which contains both $i$ and $j$ (this is equivalent by our supposition of symmetry to the statement that the sets $A_{i}$ and $A_{j}$ are not disjoint). The problem is to determine

$$
\min \sum_{i=1}^{n}\left|A_{1}\right|=2 F_{2}(n, k) .
$$

\section{\$1. Some Basic Inequalities, and some Asymptotic Results}

It is easy to see that if there exists a graph $G_{n}$ with $V\left(G_{n}\right)=k$ and diameter $\leqq d$, then

$$
n \leqq 1+k \frac{(k-1)^{d}-1}{k-2} .
$$

(1. 1) can be proved as follows: if $V\left(G_{n}\right)=k$ the number of points which can be reached from a given point, say, $P_{1}$ by an edge is $\leqq k$; the number of points which can be reached from $P_{1}$ by a path of length 2 is $\leqq k(k-1)$ and finally the number of points which can be reached by a path of length $d$ is $\leqq k(k-1)^{d-1}$. Thus if the graph has diameter $\leqq d$ we have

$$
(n-1) \leqq k\left(1+(k-1)+(k-1)^{2}+\ldots+(k-1)^{d-1}\right) .
$$

This proves (1.1). If both $n$ and $k$ are odd, then $G_{n}$ must contain at least one point of valency $\leqq k-1$ (because the number of points of odd valency cannot be odd); thus in this case we get

$$
n \leqq 1+(k-1) \frac{(k-1)^{d}-1}{k-2} .
$$

Note that for the graph shown by Fig. 1, equality stands in (1.2). For the graph shown on Fig. 2 (the so-called Petersen-graph) equality stands in (1, 1) with $n=10$, $k=3, d=2$. 

bound

As regards $F_{2}(n, k)$ we obtain easily the lower

$$
F_{d}(n, k) \geqq \frac{n(n-1)(k-2)}{2\left((k-1)^{4}-1\right)} .
$$

(1. 3) can be proved as follows: every edge is itself a path of length 1 ; it can be contained in at most $2(k-1)$ paths of length 2 , but in this way each path of length 2 is counted twice, thus the number of paths of length 2 cannot exceed $E\left(G_{e}\right)(k-1)$. In general each edge can be contained in at most $3(k-1)^{2}$ paths of length 3 , but in this way each path of length 3 is counted three times, thus the number of paths of length 3 cannot exceed

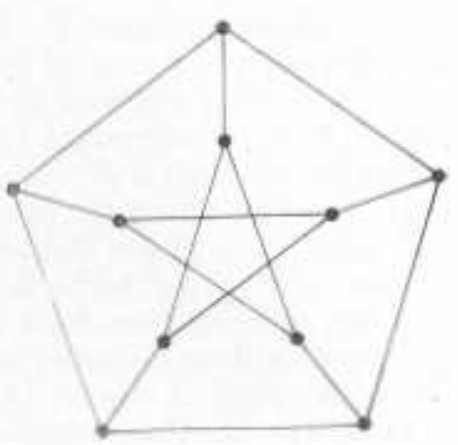

Fig. 2 $E\left(G_{n}\right)(k-1)^{2}$, etc. As in case $G_{n}$ has diameter $\geqq d$ the number of paths of length $\leqq d$ has to be at least $\left(\begin{array}{l}n \\ 2\end{array}\right)$, we obtain

$$
E\left(G_{n}\right)\left(1+(k-1)+\ldots+(k-1)^{d-1}\right) \geq\left(\begin{array}{l}
n \\
2
\end{array}\right) \text {. }
$$

which implies (1. 3). Note that one has equality in (1. 4) for the Petersen graph shown on Fig. 2., further for $n=5, k=2, d=2$ because a cycle of length 5 has 5 vertices, each of which bas valency 2 , it has diameter 2 and the number of its edges is $5=\frac{5 \cdot 4}{2 \cdot 2}$.

It is clear from the above proof that one can have equality in (1.4) only for a regular graph of order $k$, i. c. if $E\left(G_{n}\right)=\frac{n k}{2}$ and if any two points are joined by one and only one path of length $\leq d$.

The first condition implies that if equality stands in (1.4) then there is equality in (1.1) too. For the case $d=2$ this means that a necessary condition of equality in (1. 4) is $n=k^{2}+1$. It has been shown by A. J. Horfman and R. R. Singueton [4] that a regular graph of order $k$, having $k^{2}+1$ points and diameter 2 exists only for $k=2,3,7$ and perhaps for $k=57$. Thus for $d=2$ except for these values of $k$ one has strict inequality in $(1,3)$. However it has been shown in [1] that there exists an infinite sequence of pairs $\left(k_{j}, n_{j}\right)$ such that $k_{j} \rightarrow \infty, n_{j} \rightarrow \infty$ and

$$
\lim _{j \rightarrow-} \frac{F_{2}\left(n_{j}, k_{j}\right) k_{j}}{n_{j}\left(n_{j}-1\right)}=\frac{1}{2}
$$

This is a consequence of the following

THEOREM 1. If $P$ is any prime power, there exists a graph $G_{n}$ of order $n=P^{2}+P+1$ for which $V\left(G_{n}\right)=P+1$, which has diameter 2 and for which $E\left(G_{n}\right) \leqq \pm\left(n^{3 / 2}+n\right)$. The graph $G_{n}$ has also the property that it does not contain any cycle of length 4. in [1].

To make this paper self-contained we reproduce the proof of Theorem 1 given 
Proof of THEOREM 1. Let $G F(P)$ be the Galois field with $P$ elements. Let us represent the points of the finite plane geometry $P G(P, 2)$ by triples $(a, b, c)$ where $a, b, c$ are elements of $G F(P)$, not all three equal to 0 , and $(\lambda a, \lambda b, \lambda c)$ with $\lambda \in G F(P), \lambda \neq 0$ represents the same point as $(a, b, c)$. The number of different points of $P G(P, 2)$ is $P^{2}+P+1 . A$ straight line in $P G(P, 2)$ is the set of all points $(x, y, z)$ which satisfy the equation $a x+b y+c z=0$; we denote this line by $[a, b, c]$. The point $(a, b, c)$ and the line $[a, b, c]$ are clearly conjugate elements with respect to the conic $x^{2}+y^{2}+z^{2}=0$. As well known there are $P+1$ points on each line, any two different lines have exactly one point in common and through any two given points there is exactly one straight line. Now we define the mapping $T$ which maps the point $A=(a, b, c)$ into the line $\alpha=[a, b, c]$ and conversely. We write $T A=\alpha, T \alpha=A$. This mapping has evidently the properties: if the point $B$ lies on the line $\alpha=T A$ then the point $A$ lies on the line $\beta=T B$; if $C$ is the point of intersection of the lines $T A$ and $T B$ then $T C$ is identical with the line passing through the points $A$ and $B ; A=(a, b, c)$ is on $T A$ if and only if $a^{2}+b^{2}+c^{2}=0$, i.e. if $A$ lies on the conic $x^{2}+y^{2}+z^{2}=0$. Now let us define a graph $G_{n}\left(n=P^{2}+P+1\right)$ as follows: the vertices of $G_{n}$ are the points of $P G(P, 2)$; the vertices $A=(a, b, c)$ and $A^{\prime}=\left(a^{\prime}, b^{\prime}, c^{\prime}\right)$ are joined in $G_{n}$ by an edge if and only if $A^{\prime}$ is lying on $T A$ (and thus $A$ is lying on $T A^{\prime}$ ). Clearly a vertex $A$ in $G_{n}$ has the valency $P$ or $P+1$ according to whether $A$ is on the conic $x^{2}+y^{2}+z^{2}=0$ or not* Thus

$$
\frac{1}{2}\left(n^{3 / 2}-n\right) \leqq \frac{1}{2} P\left(P^{2}+P+1\right) \leqq E\left(G_{n}\right)
$$

and

$$
E\left(G_{n}\right) \leqq \frac{1}{2}(P+1)\left(P^{2}+P+1\right) \leqq \frac{1}{2}\left(n^{3 / 2}+n\right) .
$$

Finally the diameter of $G_{n}$ is equal to 2. As a matter of fact any two points $A$ and $B$ can be joined by the path $A C B$ where $C$ is the point of intersection of the lines $T A$ and $T B$. Besides this $A$ and $B$ can be joined by a single edge if $A$ lies on $T B$. But the point $C$ such that the edges $A C$ and $B C$ both belong to $G_{n}$ is in any case unique; thus $G_{n}$ does not contain any cycle of length 4 .

Thus our Theorem is proved.

We deduce from Theorem 1 the following corollaries.

Corollary 1. Put $n_{k}=k^{2}-k+1$; then

$$
\liminf _{k \rightarrow-} \frac{F_{2}\left(n_{k}, k\right) k}{n_{k}\left(n_{k}-1\right)}=\frac{1}{2} .
$$

- If $P$ is prime, there are $P+1$ points on the conic and thus

$$
E\left(G_{n}\right)=\frac{P(P+1)^{2}}{2}>\frac{1}{2} n^{3 / 2} \text { if } n \geqq n_{0} \text {. }
$$


ProOF of COROLLARY 1. By (1. 3)

$$
\frac{F_{2}(n, k) k}{n(n-1)} \geq \frac{1}{2}
$$

further if $k=P+1, n_{k}=P^{2}+P+1$, by Theorem 1

$$
F_{2}\left(P^{2}+P+1, P+1\right) \leqq \frac{1}{2}(P+1)\left(P^{2}+P+1\right) ;
$$

thus in this case

this proves our assertion.

$$
\frac{F_{2}\left(n_{k}, k\right) k}{n_{k}\left(n_{k}-1\right)} \leqq \frac{1}{2}\left(1+\frac{1}{P}\right)
$$

Theorem 1 enables us also to solve - at least asymptotically - a problem which was raised by one of us 27 years ago (see [2]).*

Let $\mathrm{C}_{n}$ denote the class of graphs having $n$ vertices and containing no cycle of order 4. Put

$$
\mu(n)=\max _{G_{n} \in \mathbf{C}_{n}} E\left(G_{n}\right) .
$$

The problem is to determine the value of $\mu(n)$. From Theorem 1 we deduce the following

Corollary 2. We have

$$
\lim _{n \rightarrow \infty} \frac{\mu(n)}{n^{3 / 2}}=\frac{1}{2}
$$

Proof of Corollary 2, It follows clearly from Theorem 1 that if $P$ is a prime power, then putting $n=P^{2}+P+1$

$$
\mu(n) \geqq \frac{1}{2}\left(n^{3 / 2}-n\right)
$$

It is possible that for these $n$ the graph of Theorem 1 is extremal but we cannot prove this. Clearly $\mu(n)$ is an increasing function of $n$, and thus it follows that for any $n$ we have

$$
\left.\mu(n) \cong \frac{1}{2}\left[P^{2}+P+1\right)^{3 / 2}-\left(P^{2}+P+1\right)\right]
$$

where $P$ is the largest prime power such that $P^{2}+P+1 \leqq n$. Now evidently for $n \geqq n_{1}$ one can choose a prime $p$ so that

$$
\sqrt{n}-\frac{\sqrt{n}}{\log n} \cong p \cong \sqrt{n}-1
$$

* After having written this paper we have been informed by W. G. BRown that independently of us he has proved (1.12), in the same way as we did. His paper will be published in the Bulletin of the Canadian Mathematical Society. 
which implies for $n \geqq n_{1}$

$$
n\left(1-\frac{2}{\log n}\right) \cong p^{2}+p+1 \leqq n .
$$

Thus we have for any $n \geq n_{1}$

$$
\mu(n) \geq \frac{1}{2} n^{3 / 2}\left(1-\frac{3}{\log n}\right)
$$

and thus

$$
\liminf _{n \rightarrow-} \frac{\mu(n)}{n^{3 / 2}} \geq \frac{1}{2} \text {. }
$$

On the other hand it is easy to see (this follows also from the results of L. REIMAN in [3]) that

$$
\limsup _{n \rightarrow-} \frac{\mu(n)}{n^{3 / 2}} \leq \frac{1}{2}
$$

As a matter of fact, let $G_{n}$ be a graph containing no cycle of order 4 , Let $P_{1}, P_{2}, \ldots, P_{n}$ be the vertices of $G_{n}$ and let us denote their valencies by $v_{1}, v_{2}, \ldots, v_{n}$. Now clearly one can select from the set $E_{i}$ of vertices joined by an edge to $P_{i}\left(\begin{array}{l}v_{i} \\ 2\end{array}\right)$ pairs, and no pair $\left(P_{j}, P_{h}\right)$ can be contained in both $E_{l}$ and $E_{l}$ with $l \neq i$ because otherwise $P_{i} P_{j} P_{l} P_{h}$ would be a cycle contained in $G_{n}$. Thus we must have

Now we have

$$
\sum_{i=1}^{n}\left(\begin{array}{l}
v_{i} \\
2
\end{array}\right) \leq\left(\begin{array}{l}
n \\
2
\end{array}\right) \text {. }
$$

and thus

$$
\left(\sum_{i=1}^{n} v_{i}\right)^{2} \cong n \sum_{i=1}^{n} v_{i}^{2}
$$

$$
\left(\sum_{i=1}^{n} v_{i}\right)^{2}-n\left(\sum_{i=1}^{n} v_{i}\right) \leqq 2 n \sum_{i=1}^{n}\left(\begin{array}{l}
v_{i} \\
2
\end{array}\right) \leqq 2 n\left(\begin{array}{l}
n \\
2
\end{array}\right) \leqq n^{3} .
$$

As clearly $\sum_{i=1}^{n} v_{i}=2 E\left(G_{n}\right)$, we have

$$
4 E^{2}\left(G_{n}\right)-2 n E\left(G_{n}\right) \leq n^{3}
$$

which implies

$$
E\left(G_{n}\right) \leq \frac{n^{3 / 2}}{2} \sqrt{1+\frac{1}{4 n}}+\frac{n}{4} .
$$

Thus

$$
\frac{\mu(n)}{n^{3 / 2}} \equiv \frac{1}{2} \sqrt{1+\frac{1}{4 n}}+\frac{1}{4 \sqrt{n}} .
$$

which implies (1. 18). Thus Corollary 2 is proved. 
Let us note that weaker results have been obtained previously by E. KLeIN (see [2]) and 1. ReImanN [3], who proved $\lim _{n \rightarrow-}$ inf $\frac{\mu(n)}{n^{3 / 2}} \geqq \frac{1}{2 \sqrt{2}}$. ReImanN's extremal graph does not contain triangles either; it is possible that among such graphs it is optimal.

Note that for the pairs $\left(n_{j}, k_{j}\right)$ for which according to Corollary 1 one has

$$
\lim _{j \rightarrow-\infty} \frac{F_{2}\left(n_{j}, k_{j}\right) k_{j}}{n_{j}\left(n_{j}-1\right)}=\frac{1}{2}
$$

one has $k_{j} \sim \sqrt{n_{j}}$. It was shown in [1] that there exists another sequence of pairs $\left(k_{j}, n_{j}\right)$ such that

$$
\lim _{j \rightarrow \infty} \frac{F_{2}\left(n_{j}, k_{j}\right) k_{j}}{n_{j}\left(n_{j}-1\right)}=1
$$

but for this sequence of pairs one has $\lim _{j \rightarrow-} \frac{k_{j}^{2}}{n_{j}}=+\cdots$

It remains an open question what is the value of the function $g(c)$ defined by

$$
g(c)=\liminf _{\substack{k^{2}>n \\ n \rightarrow-}} \frac{F_{2}(n, k) k}{n(n-1)}
$$

for $1<c<+\infty$; we know only that $g(c)$ is nondecreasing, $1 \leqq g(c)$ and $\lim _{c \rightarrow-\infty} g(c) \geq 1$.

\section{§2. Some Exact Results for $d=2$.}

In this $\S$ we deal with the exact value of $F_{2}(n, k)$ for $\frac{n}{2} \leqq k \leqq n-1$. Evidently, $F_{2}(n, n-1)=n-1$, because the graph $G_{n}$ in which one vertex is joined by an edge with all others, has diameter 2 , further $V\left(G_{n}\right)=n-1$ and $E\left(G_{n}\right)=n-1$. It has been shown in [1] that $F_{2}(n, n-2)=2 n-4$ (a graph $G_{n}$ with $V\left(G_{n}\right)=n-2$ and $E\left(G_{n}\right)=2 n-4$ and having the diameter 2 is shown by Fig. 3 ; another graph with the same properties is shown by Fig. 4), further that $F_{2}(n, n-3)=F_{2}(n, n-4)=$ $=2 n-5$. (The corresponding extremal graphs are shown by Figs. 5 and 6 .)

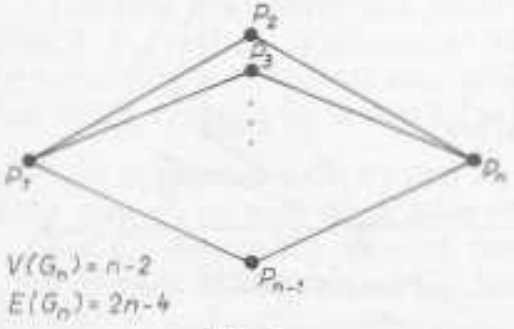

Fig. 3

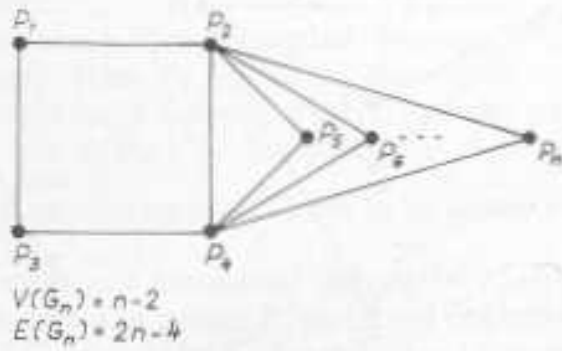

Fig, 4 
We shall prove now

THEOREM 2. We have for $n \geqq 13$

$$
F_{2}(n, k)=2 n-4 \text { for } \frac{2 n-2}{3} \leqq k \leqq n-5 \text {. }
$$

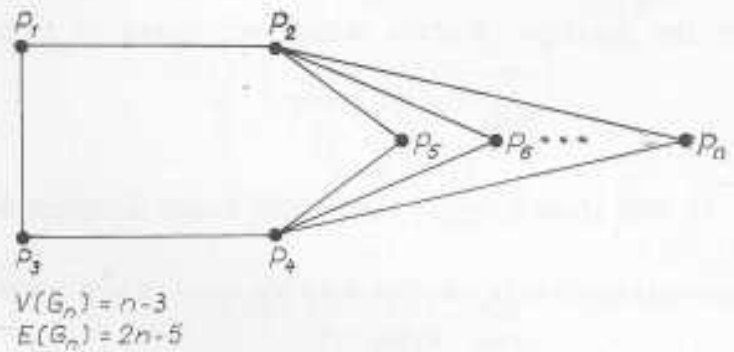

Fig. 5

Proof of THeorem 2. The extremal graph $G_{n}$ with

$$
V\left(G_{n}\right)=k=n-l, \quad\left(5 \leqq l \leqq \frac{n+2}{3}\right)
$$

and $E\left(G_{n}\right)=2 n-4$ and having diameter 2 is exhibited by Fig. 7.

$$
V\left(G_{n}\right)=n-l, \quad E\left(G_{n}\right)=2 n-4, \quad 5 \leqq l \leqq \frac{n+2}{3} ; \quad n \leqq 13 .
$$

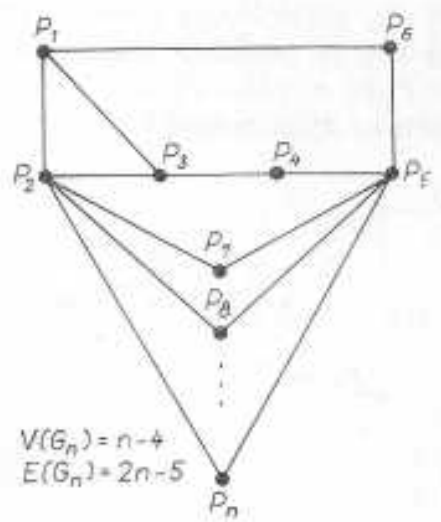

Fig. 6

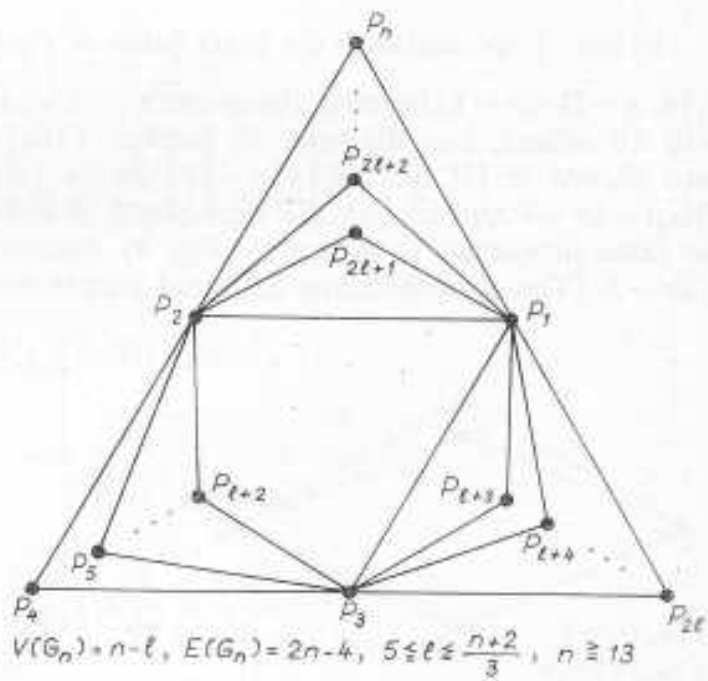

Fig. 7 
Note that all vertices of $G_{n}$ except $P_{1}, P_{2}$ and $P_{3}$ have the valency 2 , further $v\left(P_{1}\right)=n-l, v\left(P_{2}\right)=n-l, v\left(P_{3}\right)=2 l-2$ and by supposition $2 l-2 \leq n-l$. Thus $V\left(G_{n}\right)=n-L$. Clearly $G_{n}$ has diameter 2 and the number of edges of $G_{n}$ is

$$
E\left(G_{n}\right)=\frac{2(n-I)+2 I-2+2(n-3)}{2}=2 n-4 .
$$

We prove that for any $G_{n}$ with $n \geq 13, V\left(G_{n}\right)=n-I\left(5 \leq l \leq \frac{n+2}{3}\right)$ and diameter 2 one has $E\left(G_{n}\right) \geqq 2 n-4$.

As $E\left(G_{n}\right)=\frac{1}{2} \sum_{i=1}^{n} v\left(P_{i}\right)$ we may suppose that $G_{n}$ contains at least one point of degree $\leq 3$. If $G_{n}$ would contain no point of degree $\leq 2$, then let us choose a point of degree 3 ; let this point be $P_{1}$. Let the points connected by an edge with $P_{1}$ be denoted by $P_{2}, P_{3}$ and $P_{4}$. As every point can be reached from $P_{1}$ by a path of length $\leqq 2$, we must have $b\left(P_{2}\right)+v\left(P_{3}\right)+v\left(P_{4}\right) \geqslant n-1$.

Now if there would be a point among the points $P_{3}, \ldots, P_{n}$ which would be connected with more than one of the points $P_{2}, P_{3}, P_{4}$ we would have $v\left(P_{2}\right)+$ $+v\left(P_{3}\right)+n\left(P_{4}\right) \geqslant n ;$ as all other points have degree $\geq 3$ it would follow

$$
\sum_{i=1}^{n} v\left(P_{i}\right)=n+3(n-3)=4 n-9
$$

and thus $E\left(G_{n}\right)>-2 n-5$ i.e. $E\left(G_{n}\right) \geq 2 n-4$, which was to be proved. Thus we may suppose that all points $P_{1}(5 \leq i \leq n)$ are connected with one and only one of $P_{2}, P_{3}$ and $P_{4}$; similarly we can suppose that $P_{2}, P_{3}$ and $P_{4}$ are not connected with each other because this would again imply $v\left(P_{2}\right)+v\left(P_{3}\right)+v\left(P_{4}\right) \geq n$ and thus $E\left(G_{n}\right) \geqslant$ $\approx 2 n-4$. If there is at least one among the $P_{i}$ with $5 \leqq i \leqq n$ which has degree $>-3$, it again follows that $E\left(G_{n}\right) \geq 2 n-4$. If however all have degree 3 , let us suppose that $v\left(P_{2}\right)=\min \left(v\left(P_{2}\right), v\left(P_{3}\right), v\left(P_{4}\right)\right)$ which implies $v\left(P_{2}\right) \leq \frac{n-1}{3}$. Let $P_{5}$ be connected with $P_{2}$. Then $v\left(P_{5}\right)=3$ and let the three points connected with $P_{5}$ be $P_{2}, P_{i}$ and $P_{j}$; clearly $i>5$ and $j>i>5$. But then $v\left(P_{2}\right)+v\left(P_{j}\right)+v\left(P_{j}\right) \geqq n-1$ and thus

that is $n \leqq 10$.

$$
6=v\left(P_{j}\right)+v\left(P_{j}\right) \geqq \frac{2(n-1)}{3}
$$

As we supposed $n \geq 13$, this case is settled.

The case when there is a point $P_{l}$ of valency 1 is easily settled, because if this point is $P_{1}$, and $P_{1}$ is connected with $P_{2}$ only, then $P_{2}$ has to be connected with the remaining $n-2$ points too, and thus would have valency $n-1$. Thus the only case which remains to be settled is when $\min _{1=i=n} v\left(P_{i}\right)=2$. Suppose $v\left(P_{1}\right)=2$ and let $P_{1}$ be connected with $P_{2}$ and $P_{3}$. Then all remaining points have to be connected either with $P_{2}$ or with $P_{3}$ or with both.

Let $C_{1}$ denote the class of points $P_{3}$ with $i \geq 4$ connected only with $P_{2}$, and $c_{1}$ the number of elements of $C_{1}$; let $C_{2}$ be the class of points $P_{1}$ with $i \geq 4$ connected only with $P_{3}$ and $c_{2}$ the number of elements of $C_{2}$; finally let $C_{3}$ be the class of points connected with both $P_{2}$ and $P_{3}$, and $c_{3}$ the number of elements of $C_{3}$. Clearly 
$c_{1}+c_{2}+c_{3}=n-3$. As the valency of $P_{3}$ cannot exceed $n-1$ and $P_{3}$ is connected with every point in $G_{n}$ except itself and the points in $C_{1}$, we have $c_{1} \geq I-2 \geq 3$. Similarly $c_{2} \geqq l-2 \geq 3$.

The number of edges in $G_{n}$ the existence of which is already established is clearly $c_{1}+c_{2}+2 c_{3}+2=n+c_{3}-1$. Let us call these the edges of the first kind, and the remaining edges those of the second kind. As the graph has diameter 2, every point of $C_{1}$ has to be connected by a path of length $\leqslant 2$ with every point of $C_{2}$. Such a path can not contain an edge of the first kind. Thus the graph $G^{\prime}$ consisting of the edges of the second kind has to be connected. Now three cases are possible. Either $G^{\prime}$ contains besides the points of $\mathbf{C}_{1}$ and $\mathbf{C}_{2}$ at least one further point from the class $\mathbf{C}_{3}$; in this case it contains at least $c_{1}+c_{2}+1$ points and thus there are at least $c_{1}+c_{2}$ edges of the second kind, and thus the total number of edges is $E\left(G_{n}\right) \geqq n+c_{3}-1+c_{1}+c_{2}=2 n-4$. Or $P_{2}$ and $P_{3}$ are connected by an edge; in this case we get again $E\left(G_{n}\right) \geqq 2 n-4$. Or $P_{2}$ and $P_{3}$ are not connected and $G^{\prime}$ consists only of the points of $\mathbf{C}_{1}$ and $\mathbf{C}_{2}$. In this case the connected graph $G^{\prime}$ is either a tree or not. If it is not a tree, it contains at least $c_{1}+c_{2}$ edges and thus we obtain again $E\left(G_{n}\right) \geq 2 n-4$. If $G^{\prime}$ is a tree, it must have at least two end-points. We may suppose that $C_{1}$ contains an endpoint of $G$. Let $x$ be the total number of endpoints of $G^{\prime}$ in $C_{1}$. Then the sum of valencies (in $G^{\prime}$ ) of the points of $C_{1}$ is at least $x+2\left(c_{1}-x\right)$. As $G_{n}$ has diameter 2 and $P_{2}$ and $P_{3}$ are not directiy connected, any endpoint of $G^{\prime}$ in $C_{1}^{\prime \prime}$ has to be connected by a path of length 2 to $P_{3}$, it follows that for every endpoint $P$ of $G^{\prime}$ in $\mathbf{C}_{1}$ the single edge starting from $P$ ends in $\mathbf{C}_{2}$. Let $y$ denote the number of points in $C_{2}$ which are connected with an endpoint of $G^{\prime}$ in $\mathrm{C}_{1}$. If $Q$ is such a point, clearly $Q$ has to be connected with every other point of $C_{2}$, because otherwise there would not exist a path of length 2 from $P$ to these points. Now clearly no point of $C_{2}$ can be an endpoint of $G^{\prime}$, because it must be connected to at least one point in $\mathbf{C}_{1}$ and also to $Q$. Thus the sum of valencies in $G^{\prime}$ of the points of $\mathbf{C}_{2}$ ist at least $2\left(c_{2}-y\right)+y\left(c_{2}-1\right)+x$. It follows that the number of edges of the second kind is at least

$$
\frac{1}{2}\left(x+2\left(c_{1}-x\right)+2\left(c_{2}-y\right)+y\left(c_{2}-1\right)+x\right)=c_{1}+c_{2}+y\left(\frac{c_{2}-3}{2}\right) \geqq c_{1}+c_{2},
$$

because, as we have shown, $c_{2} \geqq 3$.

Thus we have shown that $E\left(G_{n}\right) \geq 2 n-4$ and the proof of Theorem 2 is complete.

Note that the restriction $n \geqslant 13$ in Theorem 2 is necessary, because for $n<13$ there is no value of $k$ between $\frac{2 n-2}{3}$ and $n-5$.

As regards the value of $F_{2}(n, k)$ for $k<\frac{2 n-2}{3}$ we can show that for $n \geq 15$

$$
F_{2}(n, k)= \begin{cases}3 n-k-6 & \text { for } \quad \frac{3 n-3}{5} \leq k<\frac{2 n-2}{3} \\ 5 n-4 k-10 & \text { for } \frac{5 n-3}{9} \leqq k<\frac{3 n-3}{5} \\ 4 n-2 k-13 & \text { for } \frac{n+1}{2} \cong k<\frac{5 n-3}{9} .\end{cases}
$$


We give in what follows the extremal graphs for these 3 cases. That these are really extremal can be proved in a way similar to the proof of Theorem 2, therefore we leave the details to the reader.

THE EXTREMAL GRAPH FOR $\frac{3 n-3}{5} \leqq k<\frac{2 n-2}{3}$.

The graph has four points of high degree; let us denote them by $A, B, C, D$ and four groups of points.

There is a group denoted by $A B$, the points of which are joined to $A$ and to $B$. The group contains $2 k-n$ points. In the group $B C D$ (connected with $B, C$ and $D$ ) there are $n-k-1$ points. In the group $A C$ (whose points are connected with $A$ and $C$ ) there are $\left[\frac{n-k-3}{2}\right]$ points; finally in the group $A D$ (the points of which are connected with $A$ and $D$ ) there are $n-k-3-\left[\frac{n-k-3}{2}\right]$ points. Further the graph contains the edges $A B, A C, A D$. The points $A$ and $B$ have the degree $k$. The whole graph has $3 n-k-6$ edges.

THE EXTREMAL GRAPH FOR $\frac{5 n-3}{9} \leqq k<\frac{3 n-3}{5}$.

There are 5 points of high order, $A, B, C, D, E$.

The group $A B$ has $2 k-n$ points,

The group $B C D$ has $\left[\frac{n-k-1}{2}\right]$ points.

The group $B C E$ has $n-k-1-\left[\frac{n-k-1}{2}\right]$ points.

The group $A C$ has $2 k-n$ points.

The group $A D E$ has $2 n-3 k-4$ points.

Further the edges $A B, A C, A D, A E, D E$ belong to the graph. The points $A, B$ and $C$ have the valency $n-k$; the total number of edges is $5 n-4 k-10$.

THE EXTREMAL GRAPH FOR $\frac{n+1}{2} \leqq k<\frac{5 n-3}{9}$.

There are 6 points of high order, $A, B, C, D, E, F$.

The group $A B$ contains $2 k-n$ points.

The group $B C E$ contains $\left[\frac{n-k-1}{2}\right]$ points.

The group $B D F$ contains $n-k-1-\left[\frac{n-k-1}{2}\right]$ points.

The group $A D C$ contains $\left[\frac{n-k-5}{2}\right]$ points.

The group AEF contain $n-k-5-\left[\frac{n-k-5}{2}\right]$ points.

The graph contains further the edges $A B, A C, A D, A E, A F$. The graph has $4 n-2 k-13$ edges. 
For $k<\frac{n+1}{2}$ we cannot determin: $F_{2}(n, k)$ cxactly. However, we can get a fairly good upper bound by constructing graphs of diameter 2 by the following principles. We divide all but $\left(\begin{array}{l}r \\ 2\end{array}\right)$ of the points of a graph $G_{n}$ into $r$ groups of approximately the same size. We connect the points of each pair of groups with one of the remaining points, and connect as many of these point; with each other

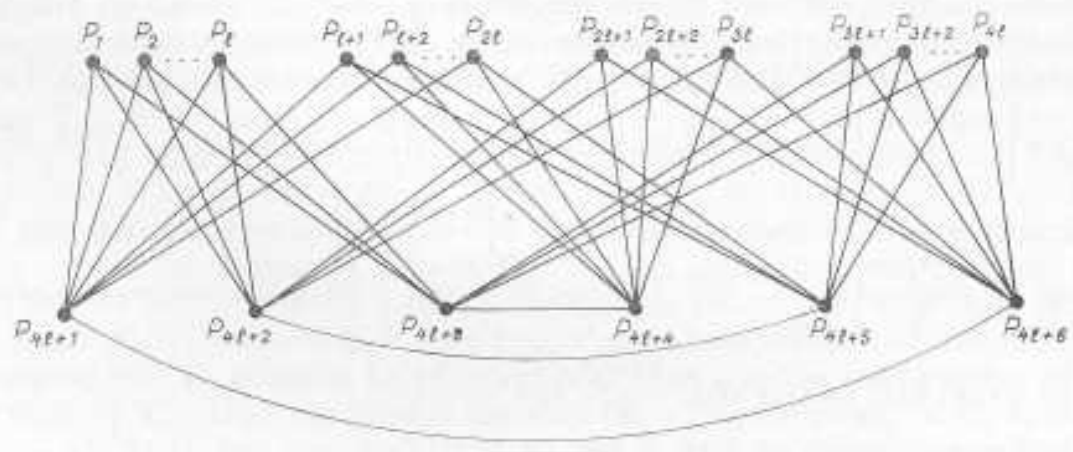

Fig. 8

as needed. For instan $z e$ if $r=4, n=4 l+6$, we put $l$ points in each of 4 groups, connect each of the 6 pairs of groups with one of the remaining 6 points, and connect each of these points with that point which is connected with the other two groups. The graph obtained is shown by Fig. 8 . It follows that

$$
F_{2}(4 l+6,2 l+1) \leqq 12 l+3 .
$$

We prove first

\section{§3. Some Results for $d \succeq 3$.}

THEOREM 3. We have for every $n$, every $k \leqq n-1$ and $d \cong 3$

$$
F_{d}(n, k) \geqq \frac{n^{2}}{k^{d-1}}\left(1-4 \sqrt[3]{\frac{n}{k^{4}}}\right) .
$$

ProOF of Theorem 3. Let us put

$$
\delta=4 \sqrt[3]{\frac{n}{k^{d}}} .
$$

Clearly we may suppose $\delta<1$, because otherwise (3. 1) is trivially fulfilled. We have evidently

$$
\delta>\frac{4}{k^{d / 3}} .
$$


We may suppose $n>k^{t-1}$, because any graph $G_{n}$ with diameter $\leqq d$ is connected and thus has at least $n-1$ edges; thus $F_{1}(n, k) \geq n-1$ and if $n \geqq k^{d-1}$ the inequality (3. 1) is trivial. Thus we have to prove $(3,1)$ only for $k^{4-1}<n<\frac{k^{d}}{64}$ i.e. for $(64 n)^{1 / d}<$ $<k<n^{1 / 4-1}$. $*$

Let $G_{n}$ be a graph having $n$ vertices, diameter $d$ and such that $V\left(G_{n}\right)=k$. Let us denote by $X_{1}, \ldots, X_{n}$, those vertices of $G_{m}$ the valency of which is $<\frac{4 n}{k^{4-1} \delta}$; let $Y_{1}, \ldots, Y_{n-1}$, be the remaining vertices of $G_{m}$. We have clearly

Thus if

$$
E\left(G_{n}\right)=\frac{1}{2}\left(\sum_{i=1}^{n} v\left(X_{i}\right)+\sum_{j=1}^{n-\pi} v\left(Y_{j}\right)\right)>\frac{2(n-s) n}{k^{d-1} \delta} .
$$

we have

$$
s \pm n\left(1-\frac{\delta(1-\delta)}{2}\right)
$$

$$
E\left(G_{n}\right) \equiv \frac{n^{2}}{k^{d-1}}(1-\delta)
$$

Thus we have to consider only the case

$$
s>n\left(1-\frac{\delta(1-\delta)}{2}\right) \text {. }
$$

We distinguish two cases. Either every $X_{i}(1 \leqq i \leqq s)$ is connected with at least $\left(1-\frac{\delta}{2}\right) \frac{n}{k^{d-1}}$ of the vertices $Y_{j}$, or not. In the first case we have

$$
E\left(G_{n}\right) \geqq s\left(1-\frac{\delta}{2}\right) \frac{n}{k^{-1}} \geqq(1-\delta) \frac{n^{2}}{k^{d-1}} .
$$

Thus we may suppose that there is an $X_{i}$ - say $X_{1}$ - which is connected with less than $\left(1-\frac{\delta}{2}\right) \frac{n}{k^{d-1}} Y_{j}$. We shall show that this case is impossible. By supposition we can reach, starting from $X_{1}$, every vertex of $G$ by a path of length $\leqq d$. Let us consider first those paths starting from $X_{1}$, the next vertex of which is an $Y_{I}$. As $Y_{j}$ can be chosen in $<\left(1-\frac{\delta}{2}\right) \frac{n}{k^{d-1}}$ ways, and all vertices of $G_{n}$ have valency $\leq k$, the number of such pathes is at most

$$
\left(1-\frac{\delta}{2}\right) \frac{n}{k^{2-1}}\left(1+(k-1)+(k-1)^{2}+\ldots+(k-1)^{k-1}\right) \leq\left(1-\frac{\delta}{2}\right) n .
$$

- We may also suppose that $k>64$. 
Let us count now the pathes of length $\leqq d$ starting from $X_{1}$, on which the point next to $X_{1}$, is an $X_{i}$. The number of such pathes is clearly at most

$$
\frac{4 n}{k^{d-1} \delta}\left(1+\frac{4 n}{k^{d-1} \delta}\left(1+(k-1)+\ldots+(k-1)^{d-2}\right)\right)<\frac{\delta n}{3} .
$$

It follows from $(3.8)$ and $(3.9)$ that the total number of vertices which can be reached from $X_{1}$ by a path of length $\cong d$, can not exceed $n\left(1-\frac{\delta}{6}\right)$ which is $\leqq n-2$ if $n \geqq \frac{12}{\delta}$, and this is true if $n-1 \geq k \geqq 64$; thus we arrived to a contradiction and this proves our theorem.

To show that the order of magnitude $\frac{n^{2}}{k^{2}}$ of the lower estimate of $F_{3}(n, k)$ is best p ossible, consider the following graph $G_{n}$ : Take a complete graph $G_{r}$ having $r$ vertices, and connect each vertex of $G_{r}$ with $r-1$ new points. Thus we obtain a graph $G_{n}$ with $r(r-1)+r=r^{2}=n$ vertices. Clearly one has $k=V\left(G_{n}\right)=2 r-2$, $D\left(G_{n}\right)=3$ and $E\left(G_{n}\right)=\frac{3}{2} r(r-1)$. Thus $E\left(G_{n}\right) \sim \frac{6 n^{2}}{k^{2}}$.

In this example $k=2(\sqrt{n}-1)$; by slightly modifying this example we obtain that

$$
F_{3}(n, k)<\frac{n^{2}}{k^{2}}(c+1)^{2}\left(\frac{1}{2}+\frac{1}{c}\right)
$$

if $k \sim c n$ where $0<c<1$.

To show that $F_{3}(n, k)$ is of order of magnitude $\frac{n^{2}}{k^{2}}$ for $k \sim \lambda \sqrt{n}$ where $0<\lambda<1$ we have to apply a more involved construction. Let us consider a graph $G_{n}$ which has the vertices $P_{g i j}$ where $1 \leqq g \leqq l, 1 \leqq i \leqq s, 1 \leqq j \leqq s$ and the vertices $Q_{\text {phi }}$ where $1 \leqq g<h \leqq l$ and $1 \leqq i \leqq s$; thus $n=l s^{2}+\left(\frac{l}{2}\right) s$. Suppose that the edges of $G_{n}$ are as follows:

a) $P_{h i j}$ and $P_{h i j}$ are both connected with $Q_{g h i}$ for $\mathrm{I} \leqq g<h \leqq l, i, j=1,2, \ldots, s$.

b) $Q_{g h i_{1}}$ is connected with $Q_{g h i_{2}}$ for $1 \leqq i_{1} \leqq s, 1 \leqq i_{2} \leqq s, i_{1} \neq i_{2}, 1 \leqq g<h \leqq l$;

c) $Q_{g_{1} h_{i} i}$ and $Q_{g_{2} h_{2} t}$ are connected for $1 \leqq g_{1}<h_{1} \leqq l$ and $1 \leqq g_{2}<h_{2} \leqq l$, $i=1,2, \ldots, s$.

Clearly

$$
\left.E\left(G_{n}\right)=2\left(\begin{array}{l}
l \\
2
\end{array}\right) s^{2}+\left(\begin{array}{l}
l \\
2
\end{array}\right)\left(\begin{array}{l}
s \\
2
\end{array}\right)+\left(\begin{array}{l}
l \\
2
\end{array}\right)\right) s
$$

further $v\left(P_{g i j}\right)=s-1$ and

$$
\left.v\left(Q_{w h i}\right)=s+1-1+\left(\begin{array}{l}
l \\
2
\end{array}\right)\right)-1
$$


and thus $V\left(G_{n}\right)=s+l+\left(\begin{array}{l}1 \\ 2 \\ 2\end{array}\right)-2$. Thus we obtain

$$
F_{3}\left(l s^{2}+\left(\begin{array}{l}
l \\
2
\end{array}\right) s, s+l+\frac{(l+1) l(l-1)(l-2)}{8}-2\right) \leqq 2\left(\begin{array}{l}
l \\
2
\end{array}\right) s^{2}+\left(\begin{array}{l}
l \\
2
\end{array}\right)\left(\begin{array}{l}
s \\
2
\end{array}\right)+\left(\begin{array}{l}
l \\
2
\end{array}\right) s .
$$

By other words by choosing for $l$ an arbitrary fixed natural number and for $s$ tending to $+\infty$, we obtain an infinite sequence of pairs $n, k$ such that

$$
k \sim \frac{\sqrt{n}}{\sqrt{l}} \text { and } F_{3}(n, k) \cong \frac{5}{4} \frac{n^{2}}{k^{2}} l(l-1) .
$$

Thus for arbitrary small $\lambda>0$ there exists an infinity of pairs $n, k$ such that $k \sim \lambda \sqrt{n}$ and

$$
F_{3}(n, k)<\frac{5}{4 \hbar^{4}} \cdot \frac{n^{2}}{k^{2}}
$$

Let us study now the behaviour of $F_{3}(n, k)$ for large values of $k$. Clearly $F_{3}(n, k)=n-1$ if $k \geqslant \frac{n}{2}$ because the graph $G_{n}$ shown on Fig. 9 has diameter 3 $V\left(G_{n}\right)=k$ and $G_{m}$ is a tree, thus it has $n-1$ edges; this result is best possible because a connected graph $G_{n}$ cannot have less than $n-1$ edges.

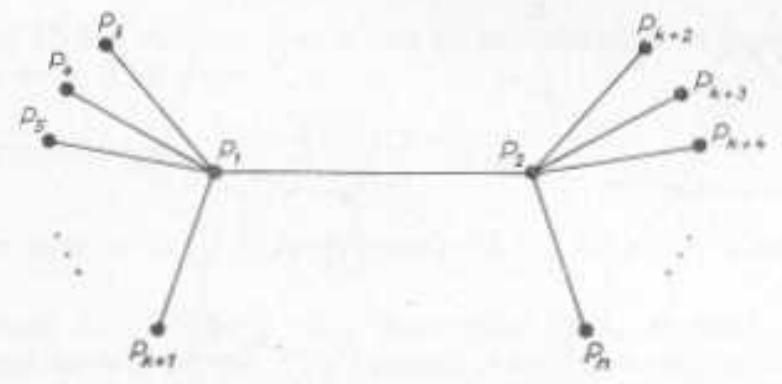

Fig. 9

We prove now the following

Theorem 4. If $\frac{n}{s+1}+s-1 \leqq k \leqq \frac{n}{s}+s-2$ where $s=1,2,3, \ldots,\left\lceil\sqrt[3]{\frac{n}{2}}\right\rfloor$ then $F_{3}(n, k)=n+\left(\begin{array}{l}s \\ 2\end{array}\right)-1$.

Proof of Theorest 4. The a ase $s=1$ has been settled above. Let us consider first the case $s=2$. Suppose $G_{s}$ would be a tree of diameter 3 and $V(G)=k \leqq \frac{n}{2}$, and let $P_{1}$ be an endpoint of $G_{n}$ (such a point exists as every tree has at least two 
endpoints). Let $P_{2}$ denote the single point connected with $P_{1}$ by an edge, and let $P_{3}, \ldots, P_{1}$ be all the other points connected with $P_{2}$; as $V\left(G_{n}\right)=k$ we have $l \leqq k+1$. The remaining $n-k-1 \geqq k-1 \geqq l-2$ points have to be connected with one of the points $P_{3}, \ldots, P_{1}$ because otherwise it would be impossible to reach them from $P_{1}$ by a path of lingth $\leqq 3$. But they can not be all connected with the same point $P_{j}(3 \leqq j \leqq I)$ because this point would have valency $>k$. Let $P_{r}$ and $P_{s}$ be two points $(l<r<s \leqq n)$ such that $P_{r}$ is connected with $P_{l}$ and $P_{\mathrm{A}}$ with $P_{f}(3 \leqq i<j \leqq l)$. Then the (unique) path from $P_{r}$ to $P_{s}$ has length 4 ; this contradiction shows that $F_{3}(n, k) \geqq n$ for $k \leqq \frac{n}{2}$.

On the other hand Fig. 10 shows a graph $G_{n}$ with $V\left(G_{n}\right)=k$ where $\frac{n}{3}+1 \leqq k \leqq \frac{n}{2}$ which has diameter 3 and contains exactly one cycle (a triangle) and thus $E\left(G_{n}\right)=n$. This completes the proof of the fact that $F_{3}(n, k)=n$ for $\frac{n}{3}+1 \leqq k \leqq \frac{n}{2}$.

Note that for $n=2 k+1$ there is another extremal graph $G_{2 k+1}$ of diameter 3, for which $V\left(G_{2 k+1}\right)=k$ and $E\left(G_{2 k+1}\right)=2 k+1$, shown by Fig. 11 .

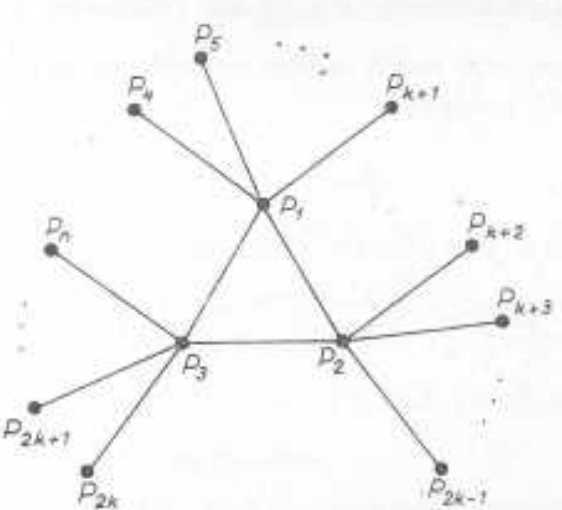

$D\left(G_{n}\right)=3, V\left(G_{n}\right)=k, E\left(G_{n}\right)=n, \frac{n}{3}+1 \leqslant k \geqq \frac{n}{2}$

Fig. 10

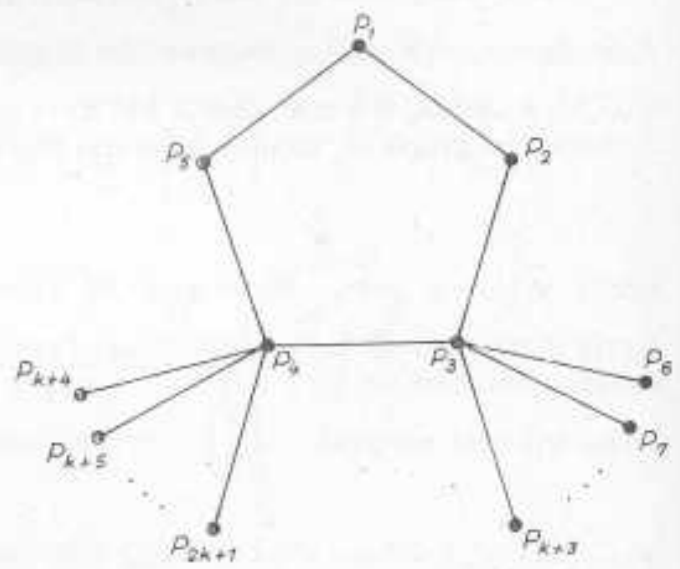

$V\left(G_{2 k+t}\right)=k, E\left(G_{2 k+t}\right)=2 k+1, D\left(G_{2 k+1}\right)=3$

Flg. 11

Now we pass to the case $s \geqq 3$.

Let $G_{n}$ be a graph with $V\left(G_{n}\right)=k\left(\frac{n}{s+1}+s-1 \leqq k \leqq \frac{n}{s}+s-2 ; s<\sqrt[3]{\frac{n}{2}}\right)$ and $D\left(G_{n}\right)=3$. Let $X_{1}, \ldots, X_{l}$ be the endpoints of $G_{n}$. As the remaining $n-l$ points all have valency $\geqq 2$, and at least one among them has valency $k$, we have

$$
E\left(G_{n}\right) \geq \frac{1}{2}(l+k+2(n-l-1))=n-\frac{l}{2}+\frac{k}{2}-1 .
$$


Now if $E\left(G_{n}\right) \geqq n+\left(\begin{array}{l}s \\ 2\end{array}\right)-1$, we have nothing to prove; if however $E\left(G_{n}\right)<n+$ $+\left(\begin{array}{l}5 \\ 2\end{array}\right)-1$ we get

$$
l>k-s(s-1) \geqq s-1
$$

thus $l \cong 2$. Let $Y_{1}, \ldots, Y_{v}$ denote those vertices of $G_{n}$ which are connected with at least one $X_{j}(i \leq j \leq l)$.

Clearly $Y_{i}$ and $Y_{j}$ are connected by an edge $(1 \leq i \leq j \leq v)$ because otherwise there would not exist a path of length 3 connecting the $X_{k}-s$. Thus it is sufficient to consider the case $v \leqq s$, because every connected graph $G$, containing a complete $s+1$-graph has at least $n-1+\left(\begin{array}{l}s \\ 2\end{array}\right)$ edges. Let us suppose therefore that $v$.s.

We prove first that $v \geqq s$. Let the endpoint $\bar{X}_{1}$ be connected to $Y_{1}$. Let $Z_{1}, \ldots Z$, denote all the points connected with $Y_{1}$ which are not endpoints of $G_{n}$. As every point of $G_{n}$ can be reached from $X_{1}$ by a path of length $\leqq 3$, if $Y_{1}$ is connected with $p$ endpoints then we have $\sum_{n=1}^{r} v\left(Z_{k}\right) \equiv n-p-1$ thus

$$
E\left(G_{n}\right) \geqq \frac{1}{2}(n-p-1+p+r+l+2(n-l-r-1))=\frac{3 n-3}{2}-\frac{r+l}{2}
$$

thus in case $E\left(G_{n}\right)<n+\left(\begin{array}{l}s \\ 2\end{array}\right)-1$ we get

$$
l \geqq n-r-s(s-1) .
$$

As however each $Y_{j}$ has valency $a k$, it can be connected to at most $k$ of the $X_{i}$ s, and $Y_{1}$ only to $k-r X_{i}$-s; thus

$$
(v-1) k+k-r \geqq n-r-s(s-1)
$$

and therefore, in view of $s \leq \sqrt[3]{\frac{n}{2}}$, we obtain $v>s-1$ i.e. $v \geq s$. Thus we have cnly to consider the case $v=s$. Now if $v=s$ there exist in $G_{n}$ at least $s$ points which are not connected to any of the $Y_{\Gamma}$ s because these bave valencies $\leqq k$ and thus the total number of points connected with them is $\leqq s(k-(s-1)) \leqq n-s$. Let $W$ be such a point.

Now clearly $W$ has to be connected with each $X_{\mathrm{h}}$ by a path of length 3 and therefore with each $Y_{j}$ by a path of length 2. Let $U_{1}, \ldots, U_{i}$ be the points connected with $W$, then each $Y_{j}$ is connected with some $U_{z}$ Thus it follows

$$
\begin{aligned}
E\left(G_{n}\right) & \geqq \frac{1}{2}(2 l+s(s-1)+2 s+2 t+2(n-l-s-t-1))= \\
& =l+\left(\begin{array}{l}
s \\
2
\end{array}\right)+s+t+n-l-s-t-1=n+\left(\begin{array}{l}
s \\
2
\end{array}\right)-1 .
\end{aligned}
$$

Thus $F_{3}(n, k) \geq n+\left(\begin{array}{l}s \\ 2\end{array}\right)-1$. On the other hand consider the graph $G_{n}$ of the following structure: let us take a complete graph $G_{x+1}$ having $s+1$ points, and connect 
each of these points except one with $k-s$ endpoints, and the last with $n-s(k-s)-$ $-(s+1)$ points. (Clearly $0 \leqq n-s(k-s)-(s+1) \leqq k-s)$.

Thus we obtain a graph $G_{n}$ with $V\left(G_{n}\right)=k, D\left(G_{n}\right)=3$ and $E\left(G_{n}\right)=n+\left(\begin{array}{l}s \\ 2\end{array}\right)-1$. This completes the proof of Theorem 4.

Let us consider now $F_{4}(n, k)$. Clearly

$$
F_{4}(n, k)=n-1 \text { if } k \geqq \sqrt{n-1} \text {. }
$$

This can be seen as follows. Fig. 12 exhibits a tree of diameter 4 showing that $F_{4}\left(k^{2}+1, k\right)=k^{2}$

Clearly if $(k-1)^{2}+1<n<k^{2}+1$, we obtain a graph $G_{n}$ exhibiting $F_{4}(n, k)=$ $=n-1$ by onvitting from the graph on Fig. $12 k^{2}+1-n$ endpoints. We shall

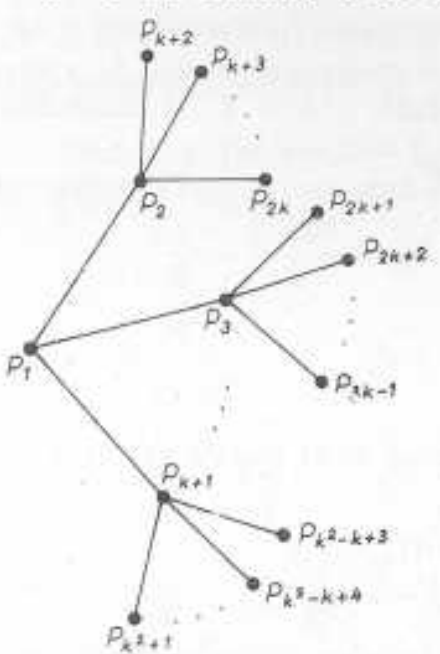

Fig. 12 prove now

THEOREM 5.

$$
F_{4}\left(k^{2}+2, k\right) \geqq k^{2}+1+\frac{1}{2} \sqrt[4]{k} \quad(k=2,3, \ldots) .
$$

ProOF OF THEOREM 5. Let $G_{k^{2}+2}$ be an extremal graph i.e, one which has $k^{2}+2$ points, diameter 4 , satisfies the condition $V\left(G_{k^{2}+2}\right)=k$ and has $F_{4}\left(k^{2}+2, k\right)$ edges.

Let $X_{1}, \ldots, X_{m}$ be the points of $G_{n^{2}+2}$ having valency $\geqq 2$, and let $G_{m}^{*}$ be the subgraph of $G_{k^{2}+2}$ spanned by these points. We assert that each point $X_{i}$ has the valency $\geq 2$ in $G_{n}^{*}$ too. Suppose that $X_{1}$ is an endpoint of $G_{m}^{*}$, and that $X_{2}$ is the only point of $G_{m}^{*}$ to which $X_{1}$ is connected. Clearly $X_{1}$ is connected with at least one endpoint $Y_{1}$ of $G_{k^{2}+2}$ because it has valency $\triangleq 2$ in $G_{k^{2}+2}$, thus it is connected with some point of $G_{k^{2}+2}$ different from $X_{2}$ and this point cannot be in $G_{m}^{*}$ and thus is an endpoint of $G_{k^{2}+2}$. Every point of $G_{k^{2}+2}$ can be reached by supposition from $Y_{1}$ by a path of length $\leqq 4$. However the number of points which can be reached from $Y_{1}$ by such a path is clearly

$$
\geqq 2 k-1+(k-1)^{2}=k^{2}
$$

which is a contradiction. Thus in $G_{m}^{*}$ each point has valency $\triangleq 2$. As the diameter of $G_{m}^{*}$ is $\leq 4$, it follows from $(1.1)$ that $G_{m}^{*}$ contains at least one point of valency $\sqrt[4]{m-1}$; thus the number of edges of $G_{m}^{*}$ exceeds $(m-1)+\frac{1}{2}(\sqrt[4]{m-1})$. Each point in $G_{m}^{*}$ can be connected with at most $k-2$ endpoints of $G_{k^{2}+2}$ thus $k^{2}+2 \leqq m+$ $+m(k-2)=m(k-1)$ and therefore $m \geqq \frac{k^{2}+2}{k-1} \geqq k+1$; thus

$$
E\left(G_{k^{2}+2}\right) \geqq k^{2}+1+\frac{1}{2}(\sqrt[4]{m-1}) \cong k^{2}+1+\frac{1}{2} \sqrt[4]{k} .
$$

Thus Theorem 5 is proved. 
Note that the statement of Theorem 5 is trivial for $k \cong 16$, because it states. only what we know already that if $D\left(G_{k^{2}+2}\right)=4$ then $G_{k^{2}+2}$ can not be a tree.

To get an upper estimate for $F_{4}\left(k^{2}+2, k\right)-k^{2}$ consider the following graph. Take a graph $G_{k+5}$ with $V\left(G_{k+5}\right)=k, D\left(G_{k+5}\right)=2$ and $E\left(G_{k+5}\right)=2 k+6$; such a graph exists according to Theorem 2 if $k \geqq 8$ (see Fig. 7 with $l=5$ ). This graph has $k+2$ points of valency 2 . Connect $k$ out of these points with $k-2$ new points each and one with $k-3$ new points. Thus we get a graph $G_{n}$ with $n=k^{2}+2$ points, such that $V\left(G_{n}\right)=k, D\left(G_{n}\right)=4$ and $E\left(G_{n}\right)=k^{2}+k+3$. Thus

$$
F_{4}\left(k^{2}+2, k\right) \leqq k^{2}+k+3 \text {. }
$$

\section{§ 4. Some further Remarks and Unsolved Problems}

First we formulate some general principles of construction which were implicitely used above.

If $G_{n}$ is a graph of diameter $d$, and such that $V\left(G_{n}\right)=k$, then if $G_{n}$ is not regular, we may construct from $G_{n}$ a graph $G_{N}$ of order $N=n+k n-E\left(G_{n}\right)$ with $V\left(G_{N}\right)=k$ and diameter $d+2$, by connecting each vertex $P_{i}$ of $G_{n}$ which has valency $v\left(P_{i}\right)<k$ with $k-v\left(P_{i}\right)$ new points. Thus

$$
F_{d+2}\left(n+k n-2 F_{d}(n, k), k\right) \leqq k n-F_{d}(n, k) .
$$

For instance we have shown that $F_{2}(n, n-5)=2 n-4$. It follows immediately from (4. 1) that

$$
F_{4}\left(n^{2}-8 n+8, n-5\right) \leqq n^{2}-7 n+4 .
$$

Notice that for each value of $d$, the extremal graphs $G_{n}$ with $V\left(G_{n}\right)=k, D\left(G_{n}\right)=d$ and having a minimal number of edges, are trees if $k$ is sufficiently large, $k \cong U_{d}(n)$ say.

We have implicitely shown that

$$
\begin{gathered}
U_{2}(n)=n-1 \\
U_{3}(n)=\frac{n}{2} \\
U_{4}(n)=\sqrt{n-1} .
\end{gathered}
$$

It can be shown that

$$
U_{5}(n)=\frac{1+\sqrt{2 n-3}}{2}
$$

further that for any fixed $s \geqq 3$ and $n \rightarrow \infty$

$$
U_{2 s}(n) \sim \sqrt{n}
$$

and

$$
U_{2 s+1}(n) \approx \sqrt{\frac{n}{2}} .
$$

The extremal tree of diameter $2 s$ has a center, while the extremal tree of diameter $2 s+1$ has a central edge. 
Notice that if $k$ decreases by one below the critical value $U_{d}(n)$, i.e. to $U_{d}(n)-1$, there is a considerable increase in the value of $F_{\mathrm{d}}(n, k)$ if $d$ is even, but not if $d$ is odd. As a matter of fact

and

$$
\begin{aligned}
& F_{2}\left(n, U_{2}(n)-1\right)-F_{2}\left(n, U_{2}(n)\right)=(2 n-4)-(n-1)=n-3 \\
& F_{3}(2 k+1, k)-F_{3}(2 k+1, k+1)=(2 k+1)-2 k=1
\end{aligned}
$$

$$
F_{3}(2 k+2, k)-F_{3}(2 k+2, k+1)=(2 k+3)-(2 k+1)=2
$$

further as proved by Theorem 5

$$
F_{4}\left(k^{2}+2, k\right)-F_{4}\left(k^{2}+1, k\right) \geq \frac{1}{2} \sqrt{k} .
$$

The situation is similar for $d>4$.

We call attention to the following problems, left open in this paper:

Proscem 1. Is the graph of Theorem 1 extremal in the sense that among all graphs with $n$ vertices and not containing any cycle of length 4 does it have the maximal number of edges? (We have proved only that it is asymptotically extremal.)

We can prove the following resuit, which is connected with Problem 1.

THEOREM 6. If $G_{n}$ is a graph in which any two points are connected by a path of length 2 and which does not contain any cycle of length 4 , then $n=2 k+1$ and

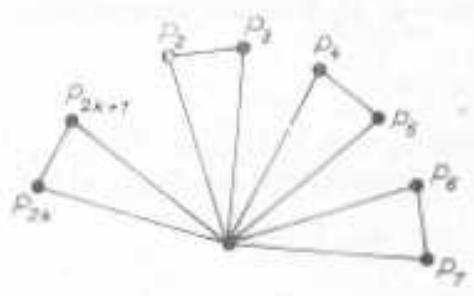

Fig. 13 $G_{n}$ consists of $k$ triangles which have one common vertex (see Fig. 13).

Proor or Theorim 6. Let $G_{n}$ be a graph with the required properties. Let $P_{1}$ be a point of $G_{n}$ having maximal valency. If $P_{1}$ is connected with all the remaining points of $G_{n}$ then evidently these have to be connected by pairs, and $G_{n}$ is of the type described in Theorem 6 . Thus we may suppose that $G_{n}$ contains at least one point $P_{2}$ which is not connected with $P_{1}$. It is easy to see that in this case $V\left(P_{2}\right)=V\left(P_{1}\right)$.

As a matter of fact there is a point $P_{3}$ in $G_{n}$ which is connected with both $P_{1}$ and $P_{2}$. As there must be a path of length 2 between $P_{1}$ and $P_{3}$ there is a point $P_{4}$ which is connected with both $P_{1}$ and $P_{3}$. As there has to be a path of length 2 between $P_{2}$ and $P_{3}$, there is a point $P_{5}$ connected with both $P_{2}$ and $P_{3}$. which is clearly different from $P_{1}, P_{2}, P_{3}$ and $P_{4}$. Let $Q_{1}, Q_{2}, \ldots, Q_{k-2}$ be the remaining points (besides $P_{3}$ and $P_{4}$ ) which are connected with $P_{1}$. Clearly $P_{2}$ and $P_{5}$ are not among the $Q_{i}$; we have $k \geq 4$ because $v\left(P_{3}\right) \geqq 4$ and by supposition $P_{1}$ has the maximal valency.

Now from each of the points $Q_{i}$ there is a path of length 2 to $P_{2}$; thus for each $Q_{i}(i=1,2, \ldots, k-2)$ there exists a point $R_{i}$ which is connected with both $Q_{i}$ and $P_{2}$. Clearly $R_{i} \neq R_{j}$ if $i \neq j$ because otherwise $G_{n}$ would contain the cycle $P_{1} Q_{i} R_{i} Q_{j}$. Further $R_{l}$ is different from $P_{3}$ because if $R_{i}$ would be identical with $P_{3} G_{n}$ would contain the cycle $P_{1} Q_{i} P_{3} P_{4}$. Finally $R_{6}$ is different from $P_{5}$ because otherwise $G_{n}$ 
would contain the cycle $P_{1} Q_{i} P_{5} P_{3}$. Thus $v\left(P_{2}\right) \geqq k$ and as $k=V\left(G_{n}\right)$ we obtain $v\left(P_{2}\right)=k=v\left(P_{1}\right)$. Thus any point of $G_{\mu}$ which is not connected with $P_{1}$ has the valency $k=v\left(P_{1}\right)$. Repeating the same argument with $P_{2}$ instead of $P_{1}$ it follows that $v\left(Q_{i}\right)=k(i=1,2, \ldots, k-2)$. As $P_{3}$ is not connected with $Q_{1}$ (because otherwise $G_{n}$ would contain the cycle $\left.P_{1} Q_{1} P_{3} P_{4}\right)$ repeating the same argument for $Q$ instead of $P_{1}$ it follows that $v\left(P_{3}\right)=k$. Thus the graph $\mathrm{G}_{n}$ is regular.

Now if $V\left(P_{i}\right)=k(i=1,2, \ldots, n)$ and $G_{n}$ does not contain a cycle of length 4 and between any two points there is a path of length 2 , then clearly if $S_{i}$ denotes the set of points connected with $P_{i}$ then the sets $S_{i}$ and $S_{j}$ have exactly one point in common, and for any two points $P_{l}$ and $P_{j}(j \neq i)$ there is exactly one point $P_{h}$ such that $S_{h}$ contains both $P_{l}$ and $P_{f}$. Thus if we define the sets of points $S_{l}$ as lines we obtain a finite plane geometry, with $k=P+1$ points on a line, and thus having $n=P^{2}+P+1$ points. But then in this geometry there would exist a one-to-one mapping beween points and lines such that no line contains the point corresponding to it, and such a mapping is known [5] to be impossible. This proves Theorem 6 .

Problem 2. To determine the exact value of $F_{2}(n, k)$ for $k<\frac{n}{2}$, or at least the asymptotic value of $F_{2}(n,[n c])$ with $0<c<\frac{1}{2}$.

Pronlem 3 . Is the lower estimate in Theorem 3 asymptotically best possible, i.e. do there exist for each $d \geqq 3$ a sequence of graphs $G_{n}(n \rightarrow \infty)$ with $V\left(G_{n}\right)=k \sim c n^{\frac{1}{d-1}}$ where $c>0$ is a constant, $D\left(G_{n}\right)=d$ and $E\left(G_{n}\right) \sim \frac{n^{2}}{k^{d-1}} \sim \frac{n}{c^{d-1}}$ ?

Problem 4. Determine asymptotically: $F_{4}\left(k^{2}+2, k\right)-k^{2}$.

Problems similar to those considered in this paper can be asked for directed graphs. We hope to return to these problems in an other paper.

(Recieived February 1, 1966.)

\section{RFFERENCFS}

(1) ERDós, P.-RENYI, A. On a problem in the theory of graphs (in Hungarian, with English and Russian summaries), Publ. Math. Inst. Hung. Acad. Sci. 7/A (1962) 623-641.

[2] ERDós, P.: On sequences of integers no one of which divides the product of two others and on some related problems, Mithellungen des Forschungsinstitutes für Math. und Mecha. nik, Tomsk, 2 (1938) $74-82$.

[3] Rerman, I.: Uber ein Problem yon K. Zarankiewicz, Acta Math. Acad. Sci. Hung. 9 (1958) $269-278$.

[4] Hoffman, A, J.-Singleton, R. R.: On Moore graphs with diameter 2 and 3, IBM Journal of Research and Development 4 (1960) 497 -504.

[5] BAER, R.; Polarities in finite projective planes, Bulletin of the American Math. Soc. $\mathbf{5 2}$ (1946) $77-93$. 